\title{
Tap changer optimisation using embedded differential evolutionary programming technique for loss control in power system
}

\author{
Ahmad Faris $^{1}$, Ismail Musirin' ${ }^{2}$, Shahrizal Jelani ${ }^{3}$, Saiful Amri Ismail, \\ Mohd Helmi Mansor ${ }^{5}$, A. V. Senthil Kumar ${ }^{6}$ \\ ${ }^{1,2,4}$ Faculty of Electrical Engineering, Universiti Teknologi MARA, Malaysia \\ ${ }^{3}$ Faculty of Engineering, Technology and Built Environment, UCSI University, Malaysia \\ ${ }^{5}$ Department of Electrical and Electronic Engineering, College of Engineering, Universiti Tenaga Nasional, Malaysia \\ ${ }^{6}$ Hindusthan College of Arts and Science, India
}

\section{Article Info}

Article history:

Received Jan 18, 2019

Revised Mar 27, 2020

Accepted Apr 14, 2020

\section{Keywords:}

Differential evolution

Embedded optimisation

Evolutionary programming

Loss control

Tap changer

\begin{abstract}
Over-compensation and under-compensation phenomena are two undesirable results in power system compensation. This will be not a good option in power system planning and operation. The non-optimal values of the compensating parameters subjected to a power system have contributed to these phenomena. Thus, a reliable optimization technique is mandatory to alleviate this issue. This paper presents a stochastic optimization technique used to fix the power loss control in a high demand power system due to the load increase, which causes the voltage decay problems leading to current increase and system loss increment. A new optimization technique termed as embedded differential evolutionary programming (EDEP) is proposed, which integrates the traditional differential evolution (DE) and evolutionary programming (EP). Consequently, EDEP was for solving optimizations problem in power system through the tap changer optimizations scheme. Results obtained from this study are significantly superior compared to the traditional EP with implementation on the IEEE 30-bus reliability test system (RTS) for the loss minimization scheme.
\end{abstract}

This is an open access article under the CC BY-SA license.

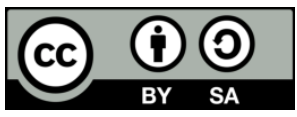

\section{Corresponding Author:}

Shahrizal Jelani,

Faculty of Engineering,

Technology and Built Environment,

UCSI University,

Kuala Lumpur, Malaysia.

Email: shahrizal@ucsiuniversity.edu.my

\section{INTRODUCTION}

The growing demand in power system network due to increasing load has caused voltage decay, leading to current increase and system loss. To curb the voltage problems, several power compensation schemes can be implemented. This requires the use of optimisation processes; among the important optimisation techniques are evolutionary programming (EP), genetic algorithm (GA) and differential evolution (DE). In the year 2008, M. Varadarajan et al. [1] reported that the method used to determine control variables needs to be varied to minimise system loss using the DE method. Several main parameters can be controlled via the parameter settings of mutation, crossover and population size. The result of system loss showed that DE provided the best solution compared to sequential quadratic programming [2]. DE is also used in order to perform parameter estimation in chaotic systems as a way to counter the issues. It also demonstrated that DE was more effective than partical swarm optimisation (PSO) and GA techniques [3]. Apart from that, 
in 2012, the study was carried out in order to minimise power losses by selecting the optimal location and parameter settings of thyristor-controlled series capacitor (TCSC).

The technique had adopted DE and GA methods, which showed that DE was more effective. Some improvements were made on DE by Z. Huang et al. [4] to establish suitable parameter adjustment method by adjusting the control parameters following the evolution stage. The results showed that DE was able to increase the ability of exploration and faster convergence speed. When a higher population is applied in $\mathrm{DE}$, the effectiveness on $\mathrm{DE}$ can be observed through the fast convergence performance [5]. Many parameters can be controlled via DE such as population size, differential weight, crossover and generation number [6]. An important study related to optimisation technique is the Optimal Power Flow (OPF). OPF is a problem that occurs in the identification of most acceptable operating levels, such as to minimise the losses and cost for electric power to make sure it satisfies the consumer demand, which flows throughout a transmission network. Loss minimisation in OPF is due to current heating [7-9].

In order to perform OPF, N. Sinha et al. $[10,11]$ found that the technique was suitable for secure and economic operation in power system. C. Ameur et al. [12] reported that any set of optimisation problems in electric power systems is known as OPF, which is one of the most practically important research subfields of constrained nonlinear optimisation. To solve the OPF problems, methods such as DE can be used for different objectives that reflect fuel cost minimisation, voltage profile improvement, and voltage stability enhancement, all of which show the effectiveness and robustness of the method used [13]. EP is useful to solving nonlinear programming problems [14]. Over the past years, OPF problems can be assessed by various techniques, such as non-linear programming, quadratic programming, mixed-integer programming and interior-point method. This types of method are categories as traditional methods. Traditional methods have some disadvantages, as they cannot be used in case of prohibited operating regions and multiple fuels [15]. In addition, they have a high sensitivity to initial solution, consequently, it may be trapped into local results, thus affecting the findings of the research. The difficulties in implementing OPF based on traditional methods can now be overcomed by modern stochastic algorithms, such as evolutionary programming (EP), tabu search (TS), improved evolutionary programming (IEP) and differential evolution (DE).

Genetic algorithm (GA) is an optimisation method for solving both constrained and unconstrained problems that are based on the natural selection of the population [16, 17]. It is commonly used to generate the best solutions for optimisation purposes by relying on the algorithm.. Tabu Search is commonly used as a mathematical optimisation tool to act as a derivative-free optimisation technique in solving OPF problems, thus significantly reducing computational burden. The main advantages of TS algorithm is its robustness to its own parameter settings. In addition, TS is characterised by its ability to avoid entrapment in local optimal solution and prevent cycling by using a flexible memory of search history [18]. Furthermore, PSO is a swarm technique developed by R. C. Eberhart et al. [19] in 1995. This technique motivated from the simulation of social behaviour. PSO, like the other optimisation techniques, is able to update the population of individuals by applying some type of operators according to the fitness information so that the population can be expected to receive better solution areas [20]. EP was also used in this study. EP is an algorithm that is works on mutation to breed the offspring [21]. In recent years, EP has been applied with success to many numerical and other optimisation problems. Nevertheless, there are weaknesses to using EP, which is slow convergence to a good near optimum [22]. EP can be compared to Genetic Algorithm (GA) in a way that EP controls the parameter and is based on mutation and selection parameters [23]. EP has two major steps in its algorithm, which are 1) mutate the solutions in the current population; and 2) select the next generation from the mutated [24]. On the other hand, J-H. Kim and H. Myung have proposed two EP methods for handling of nonlinear constrained optimisation problems [25].

This paper presents a stochastic optimization technique used to fix the power loss control in a high demand power system due to the load increase. A new technique termed as EDEP is developed to solve optimization involving loss and voltage control. Implementation on a reliability test system produced promising results; highlighting its superiority over the traditional EP.

\section{RESEARCH METHOD}

In this section, the processes involved in this study are presented as:

\subsection{Problem formulation}

Power loss in power system is the big problem for the system. They are happened due to disturbance on voltage and current as both of them are related. This can be seen through the formula:

$$
P_{\text {loss }}=\sum_{k=1}^{N_{l}} g_{k}\left(t_{k} V_{i}\right)^{2}+V_{j}^{2}-2 t_{k} V_{i} V_{j} \cos \theta_{i j}
$$


where:

$N_{l}=$ Number of transmission line

$g_{k}=$ Conductance of line, $\mathrm{vi}$ and $\mathrm{vj}=\mathrm{voltage}$ magnitude

$\theta_{i j}=$ Voltage angle difference between busses $i$ and $j$

\subsection{Differential evolution}

DE has three main operators, which are mutation, crossover and selection. These operators are similar to the EP's operators. The efficiency of DE is based on mutation and crossover.

a. Mutation

Three different vector $\mathrm{xr} 1, \mathrm{xr} 2$ and $\mathrm{xr} 3$ were randomly selected to generate mutant vector using the following formula:

$$
v_{i}^{G}=x_{r_{1}}^{G}+F\left(x_{r_{2}}^{G} x_{r_{3}}^{G}\right), \quad r_{1} \neq r_{2} \neq r_{3} \neq i
$$

b. Crossover

Crossover is a process when a mutant vector and a parent vector are combined to create a trial vector by the following formula:

$$
u_{\mu}^{G}=\left\{\begin{array}{lr}
v_{\mu}^{G} & \text { if } r_{i} \leq C, \text { or } j=J_{i}, \\
x_{\mu}^{G} & \text { Otherwise }
\end{array} \quad j=1,2, \ldots, d\right.
$$

c. Selection

In selection, competition between the parent vectors will occur. The selection process is when the value of the trial vector is less than or equal to the parent vector, the trial vector will enter to the next generation. Otherwise, the parent vector will survive and go to the next generation [16]. This operator can be described by the following formula:

$$
x_{i}^{G+1}=\left\{\begin{array}{lr}
u_{i}^{G} & \text { if } f\left(u_{i}^{G}\right) \leq f\left(x_{i}^{G}\right), \\
x_{i}^{G} & \text { Otherwise }
\end{array}\right.
$$

\subsection{Evolutionary programming}

For EP, there are two major steps which are mutation and selection of fitness. The explanations of those steps are below:

a. Initialisation

It is a process to generate random numbers, $x_{1}^{1}, x_{2}^{2} \ldots \ldots x_{k}^{\alpha}$ where $\mathrm{k}$ is the number of variables and $\alpha$ is the number of individuals.

b. Fitness Evaluation

In this phase, fitness values are calculated using generated individuals.

c. Mutation

In mutation, offspring is created using Gaussian Mutation technique by applying this formula:

$$
x_{i+m, j}=x_{i+j}+N\left(0, B\left(x_{j \max }-x_{j \min }\right)\left(\frac{f_{\text {max }}}{f_{\min }}\right)\right.
$$

d. Combination

Combination is when the parent and offspring are combined in series (by rows). The number of rows will be doubled:

$$
\begin{aligned}
& {[\text { Combination }]=\left[\begin{array}{c}
\text { parents } \\
\text { offsprings }
\end{array}\right]} \\
& {[2 m \times n]=\left[\begin{array}{lll}
m & \times & n \\
m & \times & n
\end{array}\right]}
\end{aligned}
$$

e. Selection

This process proceeds to selecting the survivors from the combination of parent and offspring.

\subsection{Proposed embedded differential evolutionary programming (EDEP) technique}

The embedded differential evolutionary programming is combination technique that was applied in this research. The mutation formula from DE was implemented in the evolutionary programming steps to

Tap changer optimization using embedded differential evolutionary programming... (Ahmad Faris) 
produce the second fitness. The major program is EP while DE is the supporting program inserted into EP. The flowchart of the proposed EDEP technique is shown in Figure 1. Mutation and crossover are steps from differential evolution which were implemented in the EP algorithm. The steps are discussed in details in this section:

a. Initialisation

Generation of random number of location (L1, L2,..i) and random number of tap changer (tap1, tap2 ....i) are based on the EP's initialisation approach as mentioned previously. 20 individuals of each control variable are created in this process.

b. Fitness 1

Parents were created based on the 20 initial individuals using selection tap and location tap from line data.

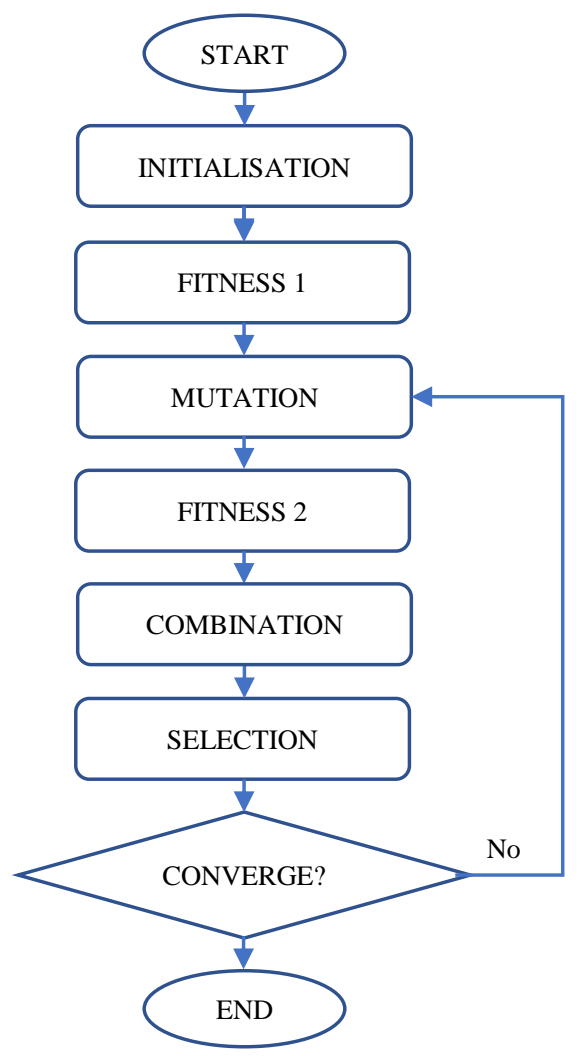

Figure 1. Proposed EDEP

c. Mutation

In this section, DE was implemented by using (2) to produce the offspring. The random number, $r$ for tap changer is selected by using a random selection $r=r a n d p e r m(20, i)$. F is a constant with a value set at 1 . To form the offspring for a new tap changer and new location, the alpha needs to be less than the crossover, which is 0.5 or the number of individuals is same to the random number that generated in the initialisation. If these conditions are not fulfilled, the previous parent value can be taken to form the tap changer and establish its location. Next, be careful for the value of location of tap changer, if the value of tap changer is less than zero, use 'abs' to form an absolute value. Then, when the location value equals to zero, take 1. Lastly, if the new location is more than maximum branch number, Ln_new>maximum branch, take the data value Ln_new=maximum branch number, because the location must be less than or equal to the data value.

d. Fitness 2

Offspring created during mutation was then used to form Fitness 2.

e. Combination

Offspring (from mutation) and parents are combined in order to perform the selection process.

f. Selection

For this section, the top 20 values for tap changer and location were selected in ascending order. 


\section{RESULTS AND DISCUSSION}

In this study, the proposed EDEP optimisation technique was implemented on the IEEE 30-bus RTS for loss minimisation process.

\subsection{Implementation of EDEP on bus 3}

Figure 2 illustrates the loss profile results using the proposed EDEP with load variation at bus 3, Qd3, which was gradually increased from 10 to 40 MVar. In general, the loss increased as the load was increased from 10 to $40 \mathrm{MVar}$. The loss profile is lower when EDEP was implemented to the system through the transformer tap changer optimisation exercise. On the other hand, Table 1 shows the results for optimal location and sizing of transformer tap setting values of load variations subjected to bus 3 . For instance, at Qd30=10 MVar, the optimal locations for tap settings were at lines 33, 32 and 36, with their corresponding optimal tap setting values of $0.5951,1.107$, and 0.6736 . The same table can be referred for other load variations.

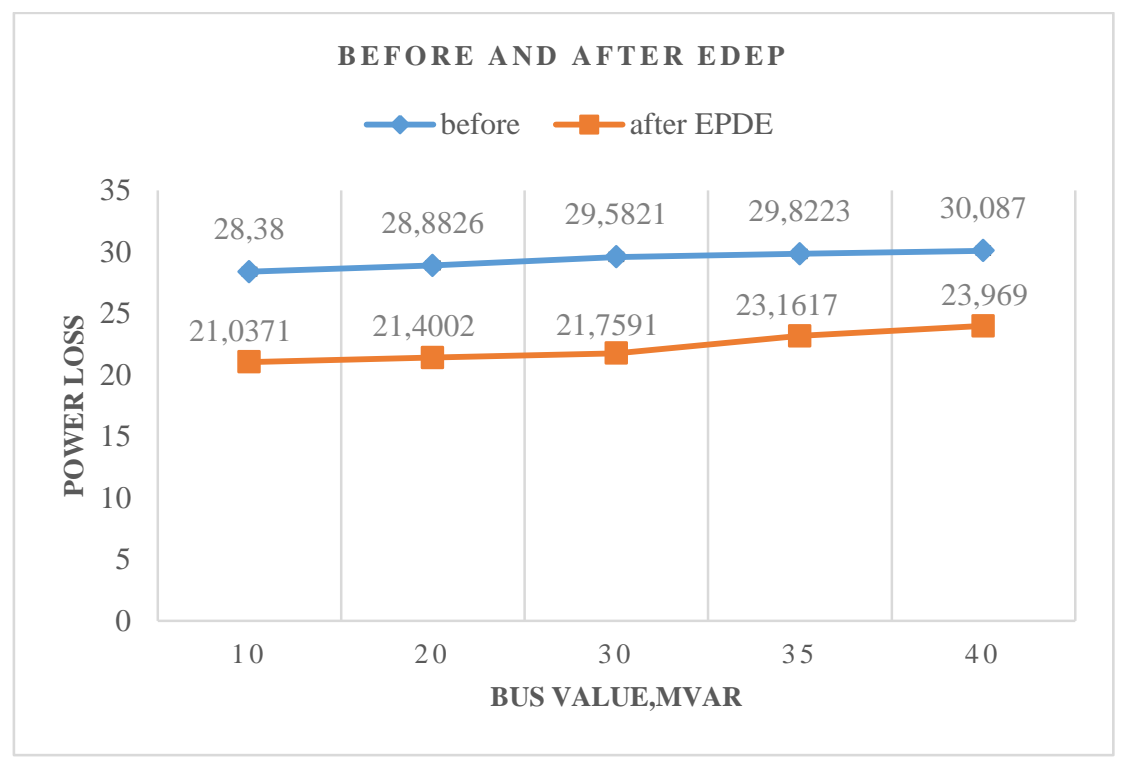

Figure 2. Result for loss profile with load variations at bus 3

Table 1. Result for optimal location and sizing of transformer taps

\begin{tabular}{llllll}
\hline & 10 MVar & 20 MVar & 30 MVar & 35 MVar & 40 MVar \\
\hline L1 & 33 & 23 & 36 & 36 & 38 \\
L2 & 32 & 41 & 9 & 16 & 36 \\
L3 & 36 & 36 & 33 & 13 & 28 \\
Tap1 & 0.5951 & 0.9763 & 0.8300 & 0.8932 & 0.9771 \\
Tap2 & 1.1070 & 1.0296 & 1.0594 & 0.9718 & 0.7956 \\
Tap3 & 0.6736 & 0.7293 & 0.9040 & 0.9800 & 0.9870 \\
\hline
\end{tabular}

\subsection{Implementation of EDEP on bus 30}

Figure 3 illustrates the result for loss profile using the proposed EDEP when the load at bus 30, Qd 30 was gradually increased from $5 \mathrm{MVar}$ to $35 \mathrm{MVar}$. In general, the loss increased as the load was increased from $5 \mathrm{MV}$ ar to $35 \mathrm{MVar}$. The loss profile is lower when EDEP was implemented to the system through the transformer tap changer optimization exercise. On the other hand, Table 2 portrays the result for optimal location and sizing of transformer tap setting values of load variations subjected to bus 3 . For instance, at $\mathrm{Qd}_{30}=5$ Mvar, the optimal location for tap settings are lines 37, 38, and 2 with their corresponding optimal tap setting values of $0.8308,0.8239$, and 0.9596 . Results for other load variations can be referred to the same table. 


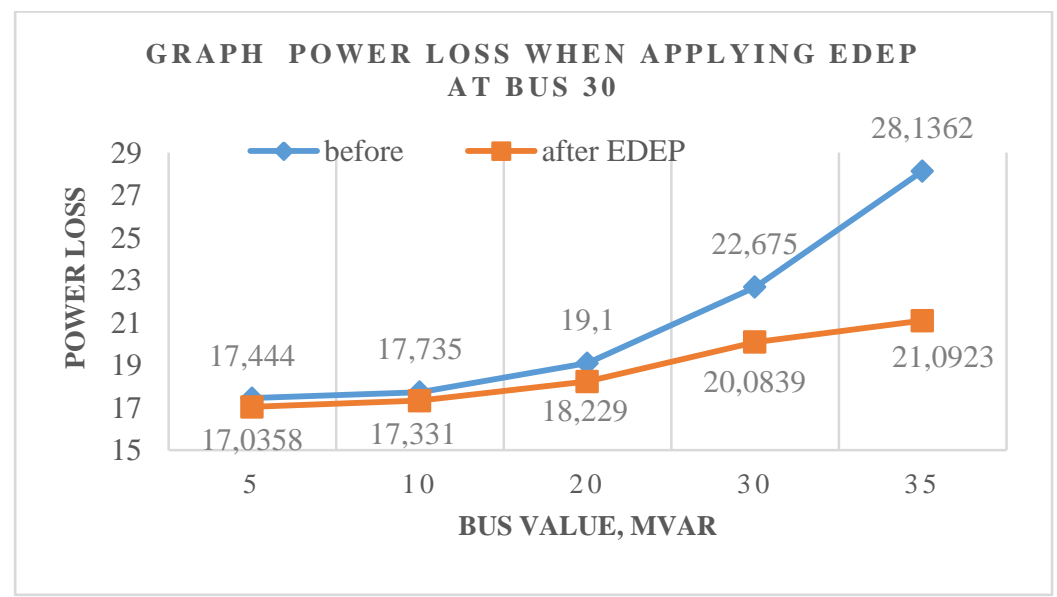

Figure 3. Result for loss profile with load variation at bus 30

Table 2. Result for optimal location and sizing of transformer taps

\begin{tabular}{llllll}
\hline & 5 MVar & 10 MVar & 20 MVar & 30 MVar & 35 MVar \\
\hline L1 & 37 & 36 & 33 & 5 & 13 \\
L2 & 38 & 14 & 3 & 36 & 11 \\
L3 & 2 & 33 & 36 & 15 & 36 \\
Tap1 & 0.8308 & 0.7658 & 0.8981 & 0.9460 & 0.9394 \\
Tap2 & 0.8239 & 0.9795 & 0.9922 & 0.7603 & 0.9128 \\
Tap3 & 0.9596 & 0.8081 & 0.8611 & 0.9629 & 0.8039 \\
\hline
\end{tabular}

\subsection{Implementation of EP}

Figure 4 illustrates the result for loss using the EP when the load at bus $30, \mathrm{Qd}_{30}$ was gradually increased from $5 \mathrm{MV}$ ar to $35 \mathrm{MVar}$. In general, the loss increased as the load was increased from $5 \mathrm{MVar}$ to 35 MVar. The loss profile is lower when EP was implemented to the system through the transformer tap changer optimization exercise. On the other hand, Table 3 demonstrates the result for optimal location and sizing of transformer tap setting values of load variations subjected to bus 3 . For instance, at $\mathrm{Qd}_{30}=5$ Mvar, optimal tap setting values are $0.9733,0.9478$, and 0.9910 . Results for other load variations can be referred to the same table.

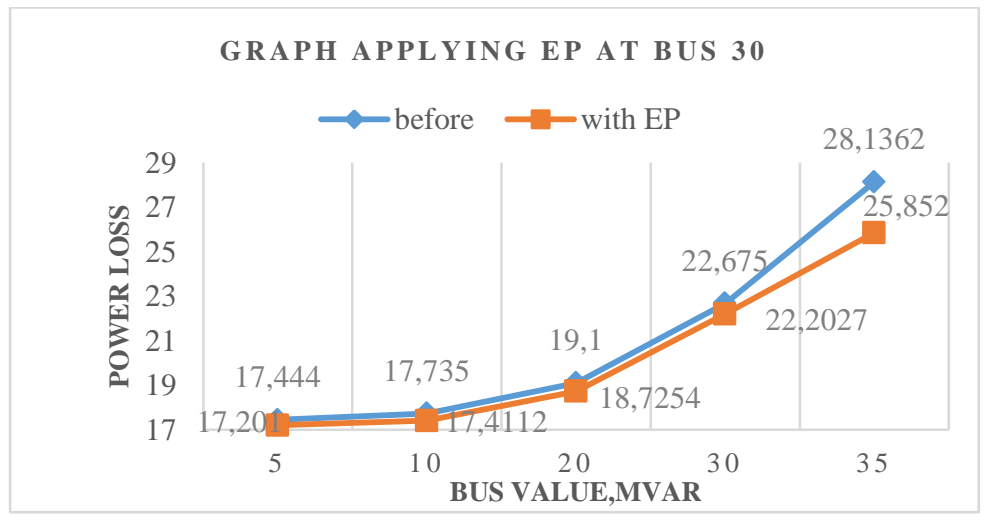

Figure 4. Result for loss profile with load variation at bus 30

Table 3. Result for optimal location and sizing of transformer taps

\begin{tabular}{llllll}
\hline & 5 MVar & 10 MVar & 20 MVar & 30 MVar & 35 MVar \\
\hline Tap1 & 0.9733 & 0.9783 & 0.9744 & 0.9533 & 0.9128 \\
Tap2 & 0.9478 & 0.9570 & 0.9588 & 0.9695 & 0.9054 \\
Tap3 & 0.9910 & 0.9926 & 0.9897 & 0.9423 & 0.9058 \\
\hline
\end{tabular}




\subsection{Comparing EDEP and EP}

Figure 5 illustrates the comparison results for loss using the proposed EDEP, EP and without optimisation where the load at bus $30, \mathrm{Qd}_{30}$ had gradually increased from $5 \mathrm{MV}$ ar to $35 \mathrm{MVar}$. In general, the loss increased as the load was increased from 5 MVar to 35 MVar. The loss profile is lower when EP and EDEP were implemented to the system through the transformer tap changer optimization exercise. The lowest loss profile that can be seen is when EDEP was implemented in this research.

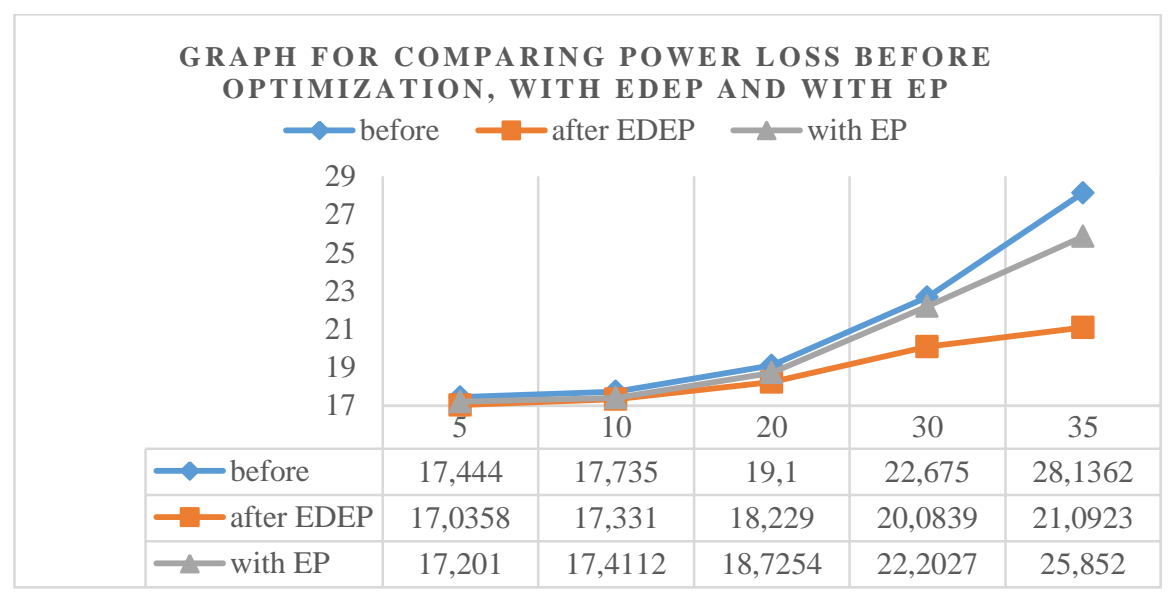

Figure 5. Result for loss profile with load variations at bus 30

\section{CONCLUSION}

A new technique was developed and termed as embedded differential evolutionary programming (EDEP) for solving optimisation problem in power system through tap changer optimisation scheme. EDEP was found to be more efficient than EP, and the mutation method from differential evolution (DE) was used to replace the Gaussian mutation method in the original EP. The comparison result between EDEP and EP proved that EDEP is the best method for power loss minimisation the loss.

\section{ACKNOWLEDGEMENTS}

The authors would like to acknowledge the UCSI University for the financial support given for this project. This research is supported by the UCSI University under the Centre of Excellence for Research, Value Innovation, and Entrepreneurship (CERVIE).

\section{REFERENCES}

[1] M. Varadarajan and K. S. Swarup, "Network loss minimization with voltage security using differential evolution," Electr. Power Syst. Res., vol. 78, no. 5, pp. 815-823, 2008.

[2] B. Peng, B. Liu, F. Y. Zhang, and L. Wang, "Differential evolution algorithm-based parameter estimation for chaotic systems," Chaos, Solitons and Fractals, vol. 39, no. 5, pp. 2110-2118, 2009.

[3] G. I. Rashed, Y. Sun, and H. I. Shaheen, "Optimal location and parameter setting of TCSC for loss minimization based on differential evolution and genetic algorithm," Phys. Procedia, vol. 33, pp. 1864-1878, 2012.

[4] Z. Huang and Y. Chen, "An improved differential evolution algorithm based on adaptive parameter," J. Control Sci. Eng., vol. 2013, pp. 1-5, 2013.

[5] B. Kazimipour, X. Li, and A. K. Qin, "Effects of population initialization on differential evolution for large scale optimization,” Proc. 2014 IEEE Congr. Evol. Comput. CEC 2014, pp. 2404-2411, 2014.

[6] J. L. Carpentier, "Optimal power flows: uses, methods and developments," IFAC Proceeding, vol. 18, no. 7, pp. 11-21, 2017.

[7] K. R. C. Mamandur and R. D. Chenoweth, "Optimal control of reactive power flow for improvements in voltage profiles and for real power loss minimization," IEEE Trans. Power Appar. Syst., vol. PAS-100, no. 7, pp. 3185-3194, 1981.

[8] J. Ma, L. Yuan, Z. Zhao, and F. He, "Transmission loss optimization-based optimal power flow strategy by hierarchical control for DC microgrids," IEEE Trans. Power Electron., vol. 32, no. 3, pp. 1952-1963, 2017.

[9] A. A. Abou El Ela, M. A. Abido, and S. R. Spea, "Optimal power flow using differential evolution algorithm," Electr. Power Syst. Res., vol. 80, no. 7, pp. 878-885, 2010. 
[10] N. Sinha, R. Chakrabarti, and P. K. Chattopadhyay, "Evolutionary programming techniques for economic load dispatch," IEEE Trans. Evol. Comput., vol. 7, no. 1, pp. 83-94, 2003.

[11] M. F. Shaari, I. Musirin, M. F. M. Nazer, S. Jaelani, F. A. Jamaludin, M. H. Mansor, and A. S. Kumar, "Supervised evolutionary programming based technique for multi-DG installation in distribution system," International Journal of Artificial Intelligent, vol. 9, no. 1, pp. 11-17, 2020.

[12] C. Ameur, S. Faquir, and A. Yahyaouy, "Intelligent optimization and management system for renewable energy systems using multi-agent," International Journal of Artificial Intelligent, vol. 8, no. 4, pp. 352-359, 2019.

[13] H. Yuan, F. Li, Y. Wei, and J. Zhu, "Novel linearized power flow and linearized OPF models for active distribution networks with application in distribution LMP," IEEE Trans. Smart Grid, vol. 9, no. 1, pp. 438-448, 2018.

[14] L. Thurner, A. Scheidler, F. Schäfer, J-H. Menke, J. Dollichon, F. Meier, S. Meinecke, and M. Braun "Pandapower-an open-source python tool for convenient modeling, analysis, and optimization of electric power systems," IEEE Trans. Power Syst., vol. 33, no. 6, pp. 6510-6521, 2018.

[15] N. S. Razali, N. Roslan, M. H. Mansor, I. Musirin, S. A. Shaaya, and S. Jelani, "Optimal reactive power control using compensating capacitor based on artificial immune system," Int. J. Adv. Trends Comput. Sci. Eng., vol. 8, no. 1.3 , pp. 381-386, 2019.

[16] G. I. Rashed, Y. Sun, and H. I. Shaheen, "Optimal location and parameter setting of TCSC for loss minimization based on differential evolution and genetic algorithm," Phys. Procedia, vol. 33, pp. 1864-1878, 2012.

[17] D. P. Kothari, "Power system optimization: Second edition," 2012 2nd National Conference on Computational Intelligence and Signal Processing (CISP), pp. 18-21, 2011.

[18] R. C. Eberhart and Y. Shi, "Comparison between genetic algorithms and particle swarm optimization," International conference on evolutionary programming. Springer, pp. 611-616, 1998.

[19] M. A. Abido, "Optimal power flow using tabu search algorithm," Electr. Power Components Syst., vol. 30, no. 5, pp. 469-483, 2002.

[20] B. Niu, H. Huang, L. Tan, and Q. Duan, "Symbiosis-based alternative learning multi-swarm particle swarm optimization,” IEEE/ACM Trans. Comput. Biol. Bioinforma., vol. 14, no. 1, pp. 4-14, 2017.

[21] H. Han, W. Lu, L. Zhang, and J. Qiao, "Adaptive gradient multi objective particle swarm optimization," IEEE Trans. Cybern., vol. 48, no. 11, pp. 3067-3079, 2018.

[22] R. C. Eberhart and Y. Shui, "Particle swarm optimization: developments, applications and resources," Proceedings of the 2001 Congress on Evolutionary Computation (IEEE Cat. No.01TH8546), vol. 1, pp. 81-86, 2001.

[23] Y. Shi and R. C. Eberhart, "Empirical study of particle swarm optimization," Proc. 1999 Congr. Evol. Comput. CEC 1999, vol. 3, pp. 1945-1950, 1999.

[24] S. A. Shaaya, I. Musirin, S. I. Sulaiman, M. H. Masor, and S. S. Mustafa, "Performance comparison of distributed generation installation arrangement in transmission system for loss control," Bulletin of Electrical Engineering and Informatics, vol. 8, no. 1, pp. 39-45, 2019.

[25] Jong-Hwan Kim and Hyun Myung, "Evolutionary programming techniques for constrained optimization problems," IEEE Trans. Evol. Comput., vol. 1, no. 2, pp. 129-140, 1997. 